\title{
MANEJO DE DESECHOS HOSPITALARIOS POR PACIENTES, FAMILIARES E INTERNAS DE ENFERMERIA DEL SERVICIO DE MEDICINA Y CIRUGÍA, HOSPITAL SANTA MARÍA DEL SOCORRO DE ICA. SETIEMBRE 2013 - SETIEMBRE 2014
}

\author{
Waste Management Hospitalarios Bypatients, Families and Nurses Internal the Department of \\ Medicine and surgery, Hospital Santa Maria Socorro of Ica. \\ September 2013 - September 2014
}

\section{Flora Martha Huisacayna Díaz ${ }^{1, a, e}$,Yolanda Aguilar Semino de Flores ${ }^{3, a, c}$, Ana Cecilia Flores García,a,a, Linda Karim Ninahuaman Huisacayna, ${ }^{2, b}$, Redu Yupanqui Cayampi ${ }^{f}$}

\author{
${ }^{1}$ Facultad de enfermería Universidad Nacional "San Luis Gonzaga" de Ica. Ica, Perú. \\ ${ }^{2}$ Facultad de Minas y Metalurgia Universidad Nacional "San Luis Gonzaga" de Ica. Ica, Perú. \\ ${ }^{3}$ Hospital Santa María del Socorro. Ica, Perú. \\ ${ }^{4}$ Hospital Augusto Hernández Mendoza. Ica, Perú. \\ ${ }^{a}$ Licenciada en Enfermería. ${ }^{b}$ Ingeniero de minas y metalurgia. ${ }^{\circ}$ Enfermera Especialista en Gerencia y Gestión de servicios de Salud . dEnfermera Especialista \\ en Pediatría. ${ }^{e}$ Magíster en Administración y Planificación de Educación Superior. 'Estudiante de enfermería
}

\begin{abstract}
Objetivo: Evaluar el manejo de los residuos sólidos Hospitalarios en las etapas del acondicionamiento por el personal de limpieza y la segregación, por los pacientes, familiares e internas de enfermería en los servicio de medicina y cirugía del Hospital Santa María del Socorro de Ica. Material y Métodos: Estudio exploratorio descriptivo longitudinal, se utilizó el tipo de muestreo no probabilístico, intencional o de conveniencia, conformado por: 60 pacientes, 90 familiares y 20 internas de Enfermería en los servicios de medicina y cirugía del Hospital Santa María del Socorro de Ica. Se utilizó como técnica la encuesta, como instrumento una guía de observación estructurada de 44 ítems el instrumento fue una guía clasificada de 5 partes La validez se determinó mediante juicios de expertos y la confiabilidad, Alfa de Cronbach de 0,79. Resultados: El acondicionamiento en los servicios de medicina y cirugía durante la primera y segunda verificación es deficiente, el proceso de segregación que realizan los pacientes es deficiente en un $100 \%$ de incumplimiento de los estándares, en los familiares es deficiente en un $100 \%$ de incumplimiento y la segregación realizada por las internas es aceptable en un 58,33\% Conclusiones: El manejo de los residuos sólidos Hospitalarios en los servicios de cirugía y medicina es deficiente.
\end{abstract}

RESUMEN

Palabras Claves: Manejo de residuos sólidos hospitalarios

\section{SUMMARY}

Objective: To assess the management of hospital waste in stages by the cleaning staff and the segregation, packaging by patients, relatives and internal of nursing in the Department of medicine and surgery of the Hospital Santa Maria of the relief of Ica. Material and methods: Iongitudinal descriptive exploratory study, we used the type of sampling non-probabilistic, intentional or convenience, consisting of: 60 patients, 90 families and 20 internal nursing in the Departments of medicine and surgery of the Hospital Santa Maria of the relief of Ica. Was used as techniqu the survey, as an instrument of observation guide structured 44-item instrument was a classified Guide to 5 parts. The validity was determined by judgments of experts and reliability, Cronbach's alpha of 0,79 . Results: Conditioning in medicine and surgery during the first and second verification services is poor; the process of segregation that patients are deficient in $100 \%$ of breach and carried out by the inmates segregation is acceptable in a 58,33\% Conclusions: the management of hospital waste in surgery and medicine services is poor.

Keywords: Management of hospital solid waste. 


\section{INTRODUCCIÓN.}

Los residuos generados en los hospitales pueden ocasionar enfermedades al personal que labora ,así como a los pacientes ,familiares y usuarios y además daño en la salud de los habitantes de la comunidad y problemas en el ambiente si el establecimiento de salud no cumple con las normas del ministerio de salud en el manejo adecuado de los desechos hospitalarios que genera El manejo de residuos en los establecimientos de salud, se encuentra con un sistema de seguridad sanitaria de ocho etapas que se inicia : Acondicionamiento, para preparar los servicios y áreas de los establecimientos de salud con el apoyo de material necesario e insumos para descartar los residuo generados ; segregación y almacenamiento primario, es decir, la separación de los residuos Sólidos generados y la eliminación en los recipientes con bolsas adecuados; Almacenamiento Intermedio acopio temporal de los residuos sólidos ; Transporte interno, traslado de los residuos a través de una ruta señalizada. Almacenamiento final, depósito de los residuos en ambiente adecuado, que no debe superar las 24 horas Tratamiento, cuyo proceso modificar las características físicas químicas o biológicas del residuo. Recolección externa, recojo de los residuos hospitalarios por parte de una empresa prestadora para la disposición final de los residuos hospitalarios. Disposición final, los residuos solido hospitalarios generados deberán ser llevados a rellenos sanitarios de acuerdo a los normas vigentes (1).

De todos los desechos que generan las actividades de atención sanitaria, aproximadamente un $80 \%$ corresponde a desechos comunes semejantes a los residuos domésticos. El restante $20 \%$ se considera material peligroso que puede ser infeccioso, tóxico o radioactivo. Se calcula que cada año se administran en el mundo 16000 millones de inyecciones, pero no todas las agujas $y$ jeringas se eliminan correctamente. Los desechos de la atención sanitaria contienen microorganismos que pueden ser dañinos e infectar a pacientes de hospital, al personal sanitario y a la población en general. En conjunto, los desechos infecciosos y de anatomía patológica constituyen el grueso de los desechos peligrosos, hasta un $15 \%$ del total de los desechos resultantes de las actividades de atención sanitaria.

Los objetos punzocortantes representan aproximadamente un $1 \%$ del total, pero cuando no se gestionan debidamente son uno de los principales vehículos de transmisión de enfermedades. Los productos químicos y farmacéuticos representan cerca de un $3 \%$ de los desechos de la atención sanitaria, mientras que los genotóxicos y los que contienen material radioactivo 0 metales pesados suponen alrededor del $1 \%$ del total (2). El personal encargado del manejo de los residuos sólidos en la etapa de segregación (generación, separación de los desechos comunes e infecciosos y almacenamiento primario en los recipientes y bolsas de color adecuado) generados en el hospital producto de la atención, recuperación diagnóstico del paciente; deben; estar entrenado adecuadamente y concientizado para cumplir con esta actividad a fin de dinamizar o eliminar el riesgo para la salud. Frente a la realidad existen; algunas debilidades en la etapa del manejo de residuos sólidos en el acondicionamiento y segregación en los servicios de estudio por los pacientes y sus familiares al realizar el almacenamiento de los residuos en recipientes no adecuados a pesar que se les educa y se les indica Es muy importante que los trabajadores pacientes y visitantes tengan conocimientos y estén capacitados en el manejo adecuado de residuos sólidos hospitalarios, debiendo ser llevados a la práctica con conciencia y cambio de actitud.

El estudio a nivel internacional por Jiménez W. en Ecuador concluye: El manejo que le dan la mayoría de los trabajadores a los residuos biológicos peligrosos generados no cumple con las normas establecidas por el 
ministerio Los graves riesgos que conlleva el manejo inadecuado de los desechos provenientes del área de la Unidad de Cuidados Intensivos (UCl) hacen imprescindible la aplicación de todas las herramientas disponibles para la prevención y control en la generación de este tipo de desechos, el $96 \%$ de enfermeras usa contenedor para objetos punzocortantes, para la eliminación de los desechos biológicos el $40 \%$ de las enfermeras hace uso del contenedor respectivo. (3).

El estudio realizado a nivel nacional por CARRIL V. Tumbes 2013: Concluye la calificación de manejo de residuos sólidos hospitalarios en el Hospital JAMO Tumbes antes del acondicionamiento califico como deficiente en la mayoría de los casos, excepto en el laboratorio que califico como aceptable y en los consultorios externos como pediatría y bienestar fetal se asignó como muy deficiente. En el hospital Carlos Cortes Jimenez- Essalud Tumbes. Alcanzo en el acondicionamiento valores de aceptable en todos los servicios, la segregación fue muy deficiente en farmacia, central de esterilización, terapia física y rehabilitación y deficiente en los demás servicios (4).

El presente trabajo proporciona datos relevantes para la institución que permitió dar a conocer, la realización el manejo de los residuos sólidos en las etapas del acondicionamiento y segregación. Como objetivo general se planteó: realizar el diagnóstico del manejo de los residuos sólidos Hospitalarios en las etapas del acondicionamiento y segregación, por los pacientes, familiares e internas de enfermería en los servicios de medicina y cirugía del hospital Santa María del Socorro de Ica.

\section{MATERIAL Y METODOS.}

El presente trabajo de investigación es de tipo cuantitativo, no experimental, de nivel exploratorio- descriptivo y diseño longitudinal. Población. La población lo constituyeron los pacientes hospitalizados en el servicio de Medicina y cirugía, familiares que acompañaron a sus pacientes durante la hospitalización de sus familiares y los internos de Enfermería que rotaron por los servicios de Medicina y cirugía durante el estudio La muestra fue de tipo de muestreo no probabilístico, intencional o de conveniencia conformada por 60 pacientes hospitalizados, 90 familiares de los pacientes hospitalizados y 20 internas de enfermería. Se utilizó como técnica la encuesta, como instrumento una guía de observación estructurada de 44 ítems, el instrumento fue una guía clasificada de 5 partes. La validez se determinó mediante juicios de expertos y la confiabilidad, Alfa de Cronbach fue de 0.79 Se utilizó el análisis estadístico descriptivo, utilizando la herramienta informática SPSS v 19,0.

\section{RESULTADOS.}

En cuanto al acondicionamiento (materiales e insumos) en los servicios de medicina y cirugía como I etapa del manejo de los residuos sólidos hospitalarios, el personal de limpieza cumple con siete (7 estándares) de dieciséis (16 estándares) equivalente al $43,7 \%$ dado un puntaje de deficiente (tabla 2); en la segunda verificación el personal de limpieza cumple con nueve (9 estándares) de dieciséis (16 estándares) equivalente al $56,2 \%$ con un puntaje de calificación de deficiente (tabla 3).

En relación al proceso de segregación (generación, clasificación y almacenamiento primario) como II etapa del manejo de los residuos sólidos hospitalarios, por los pacientes hospitalizados en los servicios de medicina y cirugía es deficiente en un $100 \%$ de incumplimiento de los ocho (8 estándares, tabla 4) verificados través de la guía de observación, la ineficacia del proceso en esta etapa aumenta el riesgo de la salud del personal, paciente, familia $y$ al deterioro ambiental

Respecto al proceso de segregación (generación, clasificación y almacenamiento primario) como II etapa del manejo de los residuos sólidos por los familiares de los pacientes hospitalizados en el servicio de 
medicina y cirugía es deficiente en un $100 \%$ de incumplimiento de (8 estándares, tabla 5) verificado a través de la guía de observación.

Referente al proceso de segregación (generación, clasificación y almacenamiento primario) como II etapa del manejo de los residuos sólidos por los internos de enfermería durante sus prácticas pre profesionales en los servicios de medicina y cirugía es aceptable en un $58,33 \%$ de cumplimiento con siete (7 estándares) y 41,7 $\%$ cinco (5 estándares) incumplidos, verificados a través de la guía de observación (tabla 6).

Tabla 1. Información sobre eliminación de los residuos sólidos hospitalarios

\begin{tabular}{lcc}
\hline INFORMACIÓN DADA A: & N & $\%$ \\
\hline Paciente & 60 & 35,3 \\
Familia & 90 & 52,9 \\
Interna de Enfermería & 20 & 11,8 \\
\hline
\end{tabular}

Tabla 2. Primera observación sobre el acondicionamiento de equipos y material para segregación de residuos hospitalarios serv. Medicina y cirugía

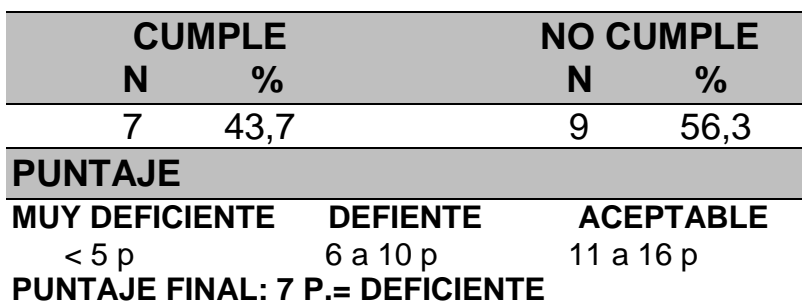

Tabla 3. Segunda observación sobre el acondicionamiento de equipos y material para segregación de residuos hospitalarios serv. Medicina y cirugía

\begin{tabular}{|c|c|c|}
\hline CUMPLE & & NO CUMPLE \\
\hline $\mathbf{N} \quad \%$ & & $\mathbf{N} \quad \%$ \\
\hline $\begin{array}{ll}9 & 56,2 \\
\end{array}$ & & 43,8 \\
\hline PUNTAJE & & \\
\hline $\begin{array}{l}\text { MUY DEFICIENTE } \\
<5 \mathrm{p}\end{array}$ & $\begin{array}{l}\text { DEFIENTE } \\
6 \text { a } 10 p\end{array}$ & $\begin{array}{l}\text { ACEPTABLE } \\
11 \mathrm{a} 16 \mathrm{p}\end{array}$ \\
\hline
\end{tabular}

Tabla4. Observación de los 8 Ítems al paciente sobre segregación de los residuos sólidos que generan en servicios de Medicina y cirugía

\begin{tabular}{ccccc}
\hline \multicolumn{2}{c}{ CUMPLE } & \multicolumn{3}{c}{ NO CUMPLE } \\
$\mathbf{N}$ & $\%$ & & $\mathbf{N}$ & $\%$ \\
\hline 0 & 00 & & 8 & 100 \\
\hline PUNTAJE & & & \\
\hline MUY DEFICIENTE & DEFIENTE & ACEPTABLE \\
$<2 \mathrm{p}$ & 3 a $6 \mathrm{p}$ & $7 \mathrm{a} 8 \mathrm{p}$
\end{tabular}

PUNTAJE FINAL: 00 P.= MUY DEFICIENTE

TABLA 5. Observación de 8 ítems a los familiares sobre segregación de los residuos sólidos que generan en los servicios de medicina y cirugía

\begin{tabular}{cccc}
\hline \multicolumn{2}{c}{ CUMPLE } & \multicolumn{2}{c}{ NO CUMPLE } \\
\hline $\mathbf{N}$ & $\%$ & $\mathbf{N}$ & $\%$ \\
0 & 0 & 8 & 100 \\
\hline \multicolumn{3}{c}{ PUNTAJE } \\
MUY DEFICIENTE & DEFIENTE & ACEPTABLE \\
$<2 \mathrm{p}$ & 3 a $6 \mathrm{p}$ & 7 a $8 \mathrm{p}$ \\
PUNTAJE FINAL: & 00 P.= MUY DEFICIENTE
\end{tabular}

TABLA 6. Observación de 8 ítems a los Internos de Enfermería sobre segregación de los residuos sólidos que generan en servicios de medicina y cirugía

\begin{tabular}{cccc}
\hline \multicolumn{3}{c}{ CUMPLE } & NO CUMPLE \\
\hline $\mathbf{N}$ & $\%$ & $\mathbf{N}$ & $\%$ \\
7 & 58,33 & 5 & 41,7 \\
\hline \multicolumn{4}{c}{ PUNTAJE } \\
\hline $\begin{array}{l}\text { MUY DEFICIENTE DEFIENTE } \\
<2 \mathrm{p}\end{array}$ & ACEPTABLE \\
< & 3 a $6 \mathrm{p}$ & 7 a $8 \mathrm{p}$ \\
PUNTAJE FINAL: 7 P.= ACEPTABLE
\end{tabular}

\section{DISCUSIÓN.}

De acuerdo con los resultados obtenidos, sobre manejo de los residuos sólidos hospitalarios en el servicio de Medicina y Cirugía ,en el acondicionamiento primera observación tabla $N^{\circ} 2$ nos muestra que cumplen con 7 ítems de dieseis equivalente al $43,7 \%$ y 9 ítems equivalente 56,35 no cumplen con el adecuado acondicionamiento por el personal de limpieza (los recipientes 
en los servicios no están etiquetados, no se cuenta con recipientes con bolsas amarillas para residuos especiales especialmente en Medicina que se administra quimioterapia, los recipientes no están cerca de la fuente de generación, no se cuenta con recipientes de volumen adecuado según las normas del Ministerio de Salud ( MINSA), el personal de limpieza no acondiciona en los servicios los recipientes para desechos punzocortantes, los recipientes para residuos punzocortantes no están rotulados, el personal de limpieza no lava los recipientes antes de colocar las bolsas respectivas ), el puntaje final fue de 7 puntos equivalente a deficiente en la primera verificación; en la segunda verificación tabla $\mathrm{N}^{\circ} 3$ nos muestra 9 ítems cumplidos con un $56,2 \%$ equivalente a deficiente, según los estándares de calificación del MINSA (6-10 puntos = deficiente). Similares resultados se encontraron en un estudio realizado por Carril V. en evaluación de los procesos de gestión ambiental de residuos sólidos región tumbes Lima, Perú 2013, quien concluye dando la calificación de deficiente en la fase del acondicionamiento en el hospital de JAMO Tumbes; el mismo autor en su estudio refiere en el hospital Carlos Cortes Jiménez Essalud Tumbes, Perú, alcanzo valores de aceptable en todos los servicios en la etapa del acondicionamiento (4). Otro estudio que difieren de los resultados por ARREVALO I. Tesis "Manejo de desechos hospitalarios por los pacientes, familiares, $86 \%$ de las personas entrevistadas opinan que existe recipientes disponibles en cantidad suficiente $y$ al alcance de los pacientes y familiares, el mismo autor contrasta los resultados en un $57 \%$ de los entrevistados indican la identificación correcta de los tachos para residuos hospitalarios. (5).

Respecto a la Segregación de los residuos sólidos de los pacientes en los servicios de Medicina y cirugía (tabla $\mathrm{N}^{\circ} 4$ ) no cumplen con la adecuada segregación y almacenamiento primaria; el $(66,7 \%)$ eliminan papel periódico, cartón, conos de papel higiénico, envolturas de medicamentos en recipientes con bolsa roja; el $83,3 \%$ elimina los residuos hasta más de las dos tercera parte del recipiente; $81,7 \%$ de los pacientes no eliminan los pañales descartables, toallas higiénicas, en los recipiente con bolsa roja ;el $66,7 \%$ de los pacientes no eliminan los restos de alimentos en los recipientes con bolsa roja; el $60 \%$ de los pacientes no elimina los papeles higiénico con secreciones en recipiente con bolsa roja; el $75 \%$ de pacientes no se lavan las manos después de eliminar los papeles higiénicos con secreciones traqueo bronquial o cuando utiliza los servicios higiénicos) ,obteniéndose el $100 \%$ de estándares no cumplidos, con un calificativo de Muy Deficiente, similares resultados se encontraron en estudios ejecutados en Ecuador por Mite V, Toclema F, Real $\mathbf{L}(6)$, quienes indican , el $67 \%$ utiliza la coloración de las fundas o bolsas de acuerdo al tipo de desecho hospitalario pero no realiza su correcta clasificación. Otro estudio similar realizado por Manchimba S, Ichau V (7), en su tesis Bioseguridad y manejo de los desechos hospitalarios por parte del personal de Enfermería del hospital San Vicente de Paul de la cuidad de Ibarra quien concluye que el personal de Enfermería no aplica correctamente la clasificación de los desechos Hospitalarios de acuerdo a las normas establecidas por el Ministerio de Salud.

En cuanto al manejo de residuos sólidos hospitalarios por los familiares en la etapa de segregación en los servicios de medicina y cirugía (tabla 5), no cumple en forma adecuado la etapa de segregación y almacenamiento mientras cuida a su paciente, el $84,4 \%$ no elimina periódicos, envolturas de papel higiénico, cartones, en los recipientes con bolsa negras, el $88,9 \%$ no elimina los residuos hasta las dos terceras partes del recipiente , el $93,4 \%$ no eliminan los pañales descartables toallas higiénicas en los recipientes con bolsa color rojo, 83,4\% no lavan las manos de su paciente después que elimine papel higiénico con secreciones o 
utiliza los servicios higiénicos el 73,4\% no elimina los residuos infecciosos de su paciente con mínimo de manipulación, el total de estándares no se cumplieron, dando un calificativo de Muy deficiente, similares resultados se encontraron en un estudio por Arévalo I. " Manejo de desechos hospitalarios por los pacientes, familiares, y personal del servicio en relación al riesgo de infecciones cruzadas en la sala de pediatría del hospital provincial general Docente Riobamba " Ecuador 2011, concluye: que existe un inadecuado manejo de desechos sólidos por parte de los pacientes, familiares que ingresan al servicio, relacionando con los resultados de la guía de observación indicando que no se cumple el $100 \%$ del manejo de los desechos hospitalarios (5).

Referente a los resultados de la guía de observación a las internas de enfermería durante el desarrollo de sus prácticas preprofesionales en el servicio de medicina y cirugía se obtuvieron (tabla 6) lo siguiente: el $55 \%$ de internos de enfermería eliminan los residuos infecciosos en los recipientes etiquetado y con bolsa de color rojo, el 65\% de los internos descarta la unidad completa jeringa y aguja en los recipientes rígidos,el 95\% de los internos de enfermería separa los desechos comunes del material corto punzante para eliminarlos ,el $70 \%$ de las internas eliminan las gasas con secreciones hemáticas, purulentas en los tachos con bolsas de color rojo, el $100 \%$ de internas no elimina los residuos punzo cortante hasta las tres cuartas partes de la capacidad del recipiente Estos resultados se contrastan con los hallazgos de Jiménez, W. en un trabajo “ Análisis del manejo de los residuos hospitalarios en el área del servicio de Medicina critica (UCl) del hospital General Luis Vernaza " Ecuador 2011 refiere : el 96\% de enfermeras hacen uso de contenedor para objetos corto punzantes, en cambio el $60 \%$ de enfermeras no hacen uso de contenedor para desechos sólidos (3).

\section{CONCLUSIONES}

1. El acondicionamiento de materiales e insumos en los servicios de medicina y cirugía por el personal de limpieza en la primera y segunda de verificación es deficiente

2. La segregación del manejo de los residuos sólidos hospitalarios por los pacientes hospitalizados en los servicios de medicina y cirugía es deficiente

3. La segregación, del manejo de los residuos sólidos hospitalarios por los familiares de los pacientes hospitalizados en los servicios de medicina y cirugía es deficiente.

4. La segregación del manejo de los residuos sólidos hospitalarios por los internos de enfermería durante sus prácticas pre profesionales en los servicios de medicina y cirugía es aceptable en un 58,33\% de cumplimiento de 12 estándares.

\section{CORRESPONDENCIA:}

Mag. Flora Martha Huisacayna Díaz

Correo electrónico: martha.fhd@hotmail.com

\section{REFERENCIAS BIBLIOGRÁFICAS}

1. Ministerio de Salud. Norma Técnica: Procedimiento para el manejo de residuo solidos hospitalarios (R. M No 212004/ MINSA) (Internet) Lima, Perú. 2004. (Citado el 26 de Enero 2014). Disponible en: http://www.disaster-info.net/PED-Suda merica/leyes/leyes/suramerica/peru/salud/ Resolucion Ministerial 217.pdf

2. Organización Mundial de la Salud (OMS). Desechos de las actividades de en atención sanitaria Centro de prensa descriptiva N²53 Nov. 2011 (Internet). Disponible en: www.who.int/mediacentre /factsheets/ fs253/es/

3. Jiménez W. "Análisis del manejo de los residuos hospitalarios en el área del servicio de Medicina Critica (uci) del hospital General Luis Vernaza" Tesis para título de Diplomado en Seguridad $Y$ salud ocupacional" Universidad Estatal de Milagro Instituto de post grado y educación Ecuador; 2011 
4. Carril V. "Evaluación de los procesos de gestión ambiental de Residuos Sólidos hospitalarios en la región de Tumbes" Tesis para optar el grado de Doctor en Ciencias Ambientales Tumbes ,Perú 2013

5. Arévalo I; Benavides, E. "Manejo de Desechos Hospitalarios por lo pacientes familiares y personal del servicio en relación al riesgo de infecciones cruzadas en la sala de pediatría del hospital del Provincial docente Riobamba "Tesis de grado previo a la obtención del título de Licenciatura en Enfermería. Universidad Estatal de Bolívar Facultad de Ciencias de la Salud- Escuela de Enfermería Riobamba, Ecuador; 2011

6. Mite V, Toclema F, Real L. Manejo de los Desechos Hospitalarios por el Personal del Centro de Salud caluma Periodo Octubre 2009-Setiembre 2010. Tesis para optar el título de Licenciada en Enfermería. Guaranda, Ecuador. Facultad de Ciencias de la Salud y del Ser Humano, Escuela de Enfermería. Universidad Estatal de Bolívar; 2010.
7. Manchimba S, Ichau V. Tesis. "Bioseguridad y manejo de Desechos Hospitalarios por parte del Personal de Enfermería del hospital San Vicente de Paul, de la cuidad de Ibarra" Tesis para título de licenciada de Enfermería Universidad Técnico del Norte. Facultad Ciencias de la Salud Escuela de Enfermería Ecuador; 2010. 